\title{
The Influence of a Hemostatic Agent on Adhesion Strength and Microleakage of Composite Resin Restorations
}

\author{
Timur V. Melkumyan, PhD, ScD*; Anjela D. Dadamova; Khaidar P. Kamilov, PhD, ScD \\ Tashkent State Dental Institute, Tashkent, Uzbekistan
}

\begin{abstract}
The aim of the present study was to assess the side effect of an aluminum chloride hemostatic agent on adhesion strength and microleakage of composite resin restorations bonded with the one-bottle total-etch adhesive system.

Methods: We prepared 10 human tooth samples (extracted premolars for orthodontic purposes) in accordance with the Ultratest technique for the assessment of shear bond strength (SBS), and another 10 human tooth samples for microleakage assessment. The SBS tooth samples $(\mathrm{n}=10)$ were subjected to the two following tests: In Test 1 , before traditional adhesive protocol, the aluminum chloride hemostatic agent was rubbed into a surface dentin for $60 \mathrm{sec}$ with the help of a metal dentoinfusor tip and washed with distilled water. In Test 2 , just traditional wet bonding was performed. In the group of teeth $(\mathrm{n}=10)$ for microleakage assessment, we prepared two round artificial cavities of similar size ( $3 \mathrm{~mm}$ in diameter, $1 \mathrm{~mm}$ deep) in each tooth sample on the proximal surfaces with half in enamel and another half in root dentin. All created cavities $(\mathrm{n}=20)$ were divided into two subgroups. In cavities of Subgroup $1(\mathrm{n}=10)$, the adhesive protocol and filling with composite resin were performed after preliminary rubbing-in of the hemostatic agent. In Subgroup $2(\mathrm{n}=10)$, cavities were merely restored according to the rules for applying the one-bottle total-etch adhesive system. Assessment of microleakage was performed at the enamel margin and dentin margin. Scanning electron microscopy was used to evaluate the microstructure morphology of a hybrid layer, formed without the side effect of a hemostatic agent and after application of it.

Results: The average score of SBS was $7.42 \pm 3.5 \mathrm{~kg}$ in Test 2 and $3.87 \pm 2.45 \mathrm{~kg}$ in Test 1 . Therefore, the side effect of the aluminum chloride hemostatic agent was detrimental to the bond strength of the composite resin to human dentin and significantly decreased the quality of adhesion by 1.92 times $(P<0.05)$. The average scores of dye penetration through the enamel-composite microgap in both subgroups were low $(0.5 \pm 0.52 \mathrm{CU}$ in Subgroup 1 and $0.3 \pm 0.48 \mathrm{CU}$ in Subgroup 2) and had no significant difference $(P>0.05)$. However, the visual analysis of the dentin-composite junction of sectioned tooth samples demonstrated 2.1 times more microleakage in Subgroup $1(1.7 \pm 0.95 \mathrm{CU})$ than in Subgroup $2(0.8 \pm 0.79 \mathrm{CU})$, but the difference was not significant $(P>0.05)$. In view of clinical situations with no possibility to escape the application of a hemostatic agent in cases of possible capillary hemorrhage and crevicular fluid leakage, it could be wise before running an adhesive protocol to cut off the portion of surface dentin that was exposed to an aluminum chloride hemostatic agent side effect. (International Journal of Biomedicine. 2018;8(4):355-357.)
\end{abstract}

Key Words: shear bond strength $\bullet$ microleakage $\bullet$ hemostatic agent $\bullet$ side effect

\section{Introduction}

The importance of composite adhesion to hard tooth tissues should not be underestimated. Cavities with close proximity to the gingiva may represent a great challenge to a clinician when placing a composite restoration. The chance of contamination of the operative field with blood and

*Corresponding author: Prof. Timur V. Melkumyan, PhD ScD. Tashkent State Dental Institute. Tashkent, Uzbekistan. E-mail: t.dadamov@gmail.com gingival crevicular fluid (GCF) is very high, even in the cases of cord gum retraction and rubber dam application. ${ }^{(1-4)}$

Aluminum chloride hemostatic agents were invented for dentists as materials of high efficacy and potency to prevent a conditioned tooth hard tissue from blood and GCF contamination. They serve restorative dentists to keep a prepared area dry and clean, which meets the needs of quality adhesive protocol. ${ }^{(5-8)}$

In accordance with the manufacturer's instructions, the hemostatic agent is for gum tissues and in some cases has to be intensively rubbed in the gums. Therefore, the effect on 
exposed dentin may be considered as a side effect of an agent or a proper contamination.

There are many studies indicating that such materials may induce changes in exposed dentin and impede the bond strength quality of a composite resin to dentin because of their acidic nature. ${ }^{\left({ }^{9-12}\right)}$ At the same time, bad composite adhesion can be the reason for low resistance to debonding stresses, which usually leads to extensive micro-leakage along the tooth-composite interface. ${ }^{(13)}$

Thus, the aim of the present study was to assess the side effect of an aluminum chloride hemostatic agent on adhesion strength (SBS) and microleakage of composite resin restorations bonded with the one-bottle total-etch adhesive system.

\section{Materials and Methods}

We prepared 10 human tooth samples (extracted premolars for orthodontic purposes) in accordance with the Ultratest technique for the assessment of shear bond strength (SBS), and another 10 human tooth samples for microleakage assessment.

The SBS tooth samples $(\mathrm{n}=10)$ were subjected to the two following tests: In Test 1, before traditional adhesive protocol, the aluminum chloride hemostatic agent (ViscoStat Clear, Ultradent) was rubbed into a surface dentin for $60 \mathrm{sec}$ with the help of a metal dento-infusor tip and washed with distilled water. In Test 2 , just traditional wet bonding was performed.

In the group of teeth $(\mathrm{n}=10)$ for microleakage assessment, we prepared two round artificial cavities of similar size $(3 \mathrm{~mm}$ in diameter, $1 \mathrm{~mm}$ deep) in each tooth sample on the proximal surfaces with half in enamel and another half in root dentin (Figure 1). All created cavities $(\mathrm{n}=20)$ were divided into two subgroups. In cavities of Subgroup $1(\mathrm{n}=10)$, the adhesive protocol and filling with composite resin were performed after preliminary rubbing-in of the hemostatic agent. In Subgroup $2(n=10)$, cavities were merely restored according to the rules for applying the one-bottle total-etch adhesive system. Assessment of microleakage was performed at the enamel margin and dentin margin.

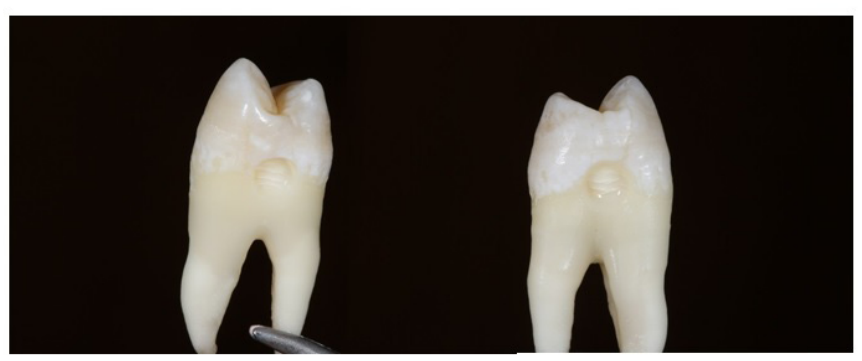

Fig. 1. Round artificial cavities $(3 \times 1 \mathrm{~mm})$ on tooth proximal surfaces.

In both the SBS and microleakage parts of the study, the adhesive protocols were the same and the bonding agent was applied in accordance with the strict indications of the manufacturer's manual for the product. The etching gel was Fine Etch-37(Spider Dent, South Korea), the adhesive was Swiss TEC SL Bond, and the composite of choice was Palfique
ESTELITE paste (Tokuyama, Japan). Light polymerization was performed with the help of Bluephase 20i (Ivoclar, Vivadent) in the "High" mode.

The SBS tooth samples were tested in the UltraTest machine at a crosshead speed of $1 \mathrm{~mm} / \mathrm{min}$ until adhesion failure occurred. Evaluations were registered in kilograms.

The microleakage tooth samples, after placement of restorations, finishing and polishing, were thermocycled, sealed with a sticky wax, and coated with two layers of nail varnish, with the exception of the restoration site and a 1 $\mathrm{mm}$ distance around of it. Tooth samples were stained in $2 \%$ methylene blue solution for 24 hours and sectioned through the centers of restorations. ${ }^{(14)}$

We evaluated dye penetration along the tooth-composite interface with a digital Canon EOS-5D camera mounted with Canon Macro Lens EF and scored on a nonparametric scale from 0 to 4 .

Randomly selected specimens, which were not subjected to microleakage evaluation, were scanned by a scanning electron microscope to evaluate the microstructure morphology of a hybrid layer, formed without the side effect of a hemostatic agent and after application of it.

Statistical analysis was performed using StatSoft Statistica v6.0. The mean (M) and standard deviation (SD) were calculated. The Mann-Whitney U Test was used to compare the differences between two groups. A probability value of $P<0.05$ was considered statistically significant.

\section{Results and Discussion}

The average score of SBS was $7.42 \pm 3.5 \mathrm{~kg}$ in Test 2 and $3.87 \pm 2.45 \mathrm{~kg}$ in Test 1 . Therefore, the side effect of the aluminum chloride hemostatic agent was detrimental to the bond strength of the composite resin to human dentin and significantly decreased the quality of adhesion by 1.92 times $(P<0.05)$.

As to the depth of microleakage (Fig.2), the average scores of dye penetration through the enamel-composite microgap in both subgroups were low $(0.5 \pm 0.52 \mathrm{CU}$ in Subgroup 1 and $0.3 \pm 0.48 \mathrm{CU}$ in Subgroup 2) and had no significant difference $(P>0.05)$. However, the visual analysis of the dentin-composite junction of sectioned tooth samples demonstrated 2.1 times more microleakage in Subgroup 1 (1.7 $\pm 0.95 \mathrm{CU})$ than in Subgroup 2 $(0.8 \pm 0.79 \mathrm{CU})$, but the difference was not significant $(P>0.05)$.

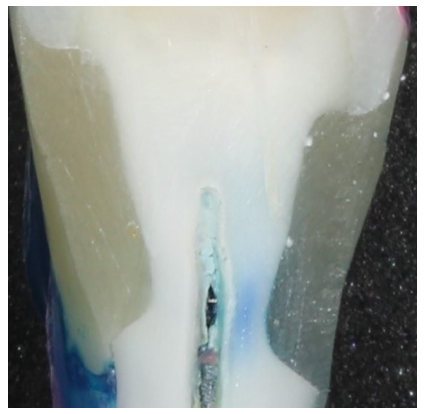

(a)

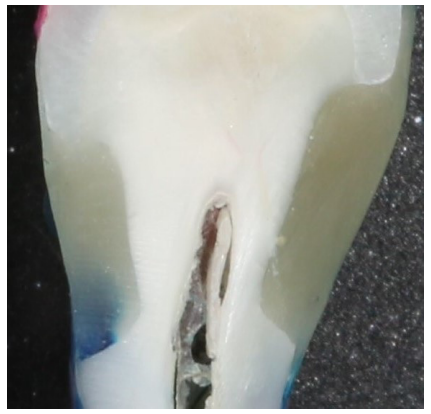

(b) (c) (d)
Fig. 2. Macroscopic images of a sectioned tooth sample with methylene blue dye penetration along the tooth-composite interface: a,d-Subgroup 2; b,c-Subgroup 1. 
One of the basic requirements for performance of a quality composite resin adhesion is a clean tooth surface to be restored or bonded. That is why much attention is paid to isolation of the bonding area for prevention of contamination in time of placement of restoration. ${ }^{(15)}$

The application of aluminum chloride hemostatic gel for bleeding and gingival crevicular fluid control is a common method of tissue management, and such agents are named by many dentists as a «can't live without product». . $^{(1,2)}$

In vitro tests on adhesion strength and microleakage of resin composites substantiated with an analysis of SEM images are very informative and may have a prognostic value for dental practice. It has been shown that the side effect of an aluminum chloride hemostatic agent on the adhesion of a composite to human dentin is rather negative and should be considered as a tooth surface contamination.

Microscopic study of a hybrid interface discovered a decreased amount and length of adhesive tags formed in dentin after the exposure to the aluminum chloride hemostatic agent (Figures 3 and 4). The last observation additionally substantiates the fact of low bond strength of composite fillings and probable microleakage along the dentin-composite interface.

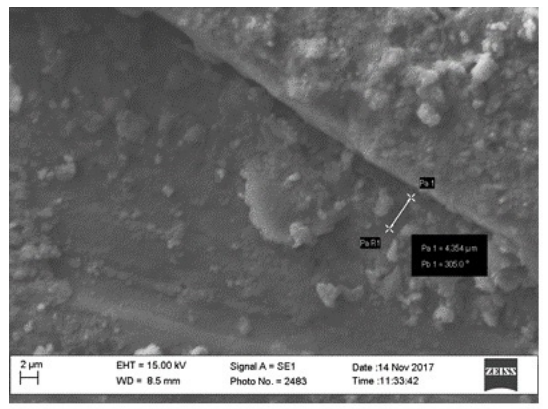

Fig. 3. SEM image of dentin-composite interface after side effect of aluminum chloride hemostatic agent.

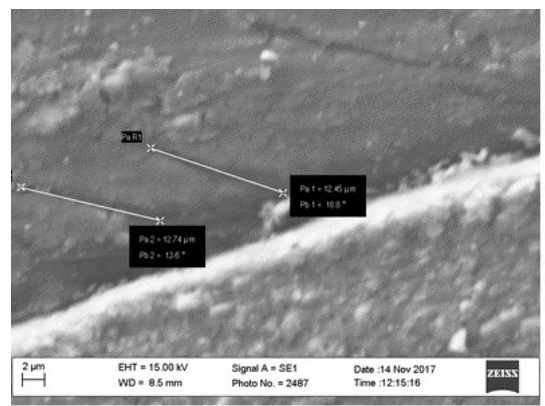

Fig. 4. SEM image of dentin-composite interface without side effect of aluminum chloride hemostatic agent.

Discrepancies in resulting significance of SBS and microleakage assessments may be due to the evident differences in character of debonding stresses used in tests. The nature of SBS examination is associated with the shear force application for registration of the level of the adhesion failure that occurred. As to the evaluation of microleakage, the detrimental effect of thermocycling may be compensated with close thermal expansion coefficients of tooth tissues and composite material.
Therefore, in view of clinical situations with no possibility to escape the application of a hemostatic agent in cases of possible capillary hemorrhage and crevicular fluid leakage, it could be wise before running an adhesive protocol to cut off the portion of surface dentin that was exposed to an aluminum chloride hemostatic agent side effect.

\section{Conflict of interest}

The authors declare that they have no competing interests.

\section{References}

1. Strassler HE, Boksman L. Tissue management, gingival retraction and hemostasis. Oral Health Journal. 2011;101:35-42.

2. Thomas MS, Joseph RM, Parolia A. Nonsurgical gingival displacement in restorative dentistry. Compend Contin Educ Dent. 2011 Jun;32(5):26-34; quiz 36, 38.

3. Kumbuloglu O, UserA, Toksavul S, Boyacioglu H. Clinical evaluation of different gingival retraction cords. Quintessence Int. 2007;38(2):e92-8.

4. Koppolu M, Gogala D, Mathew VB, Thangala V, Deepthi M, Sasidhar N. Effect of saliva and blood contamination on the bond strength of self-etching adhesive system- An in vitro study. J Conserv Dent. 2012;15(3):270-3. doi: 10.4103/0972-0707.97956. 5. Tarighi P, Khoroushi M. A review on common chemical hemostatic agents in restorative dentistry. Dent Res J (Isfahan). 2014;11(4):423-8.

6. Gupta G, Kumar S, Rao H, Garg P, Kumar R, Sharma A, et al. Astringents in dentistry: a review. Asian J Pharm Health Sci. 2012; 2: 428-432.

7. Bailey JH, Fischer DE. Procedural hemostasis and sulcular fluid control: a prerequisite in modern dentistry. Pract Periodontics Aesthet Dent. 1995; 7(4): 65-75; quiz 76.

8. de Carvalho Mendonça EC, Vieira SN, Kawaguchi FA, Powers J, Matos AB. Influence of blood contamination on bond strength of a self-etching system. Eur J Dent. 2010;4(3): 280-6. 9. Sharafeddin F, Farhadpour H. Evaluation of Shear Bond Strength of Total- and Self-etching Adhesive Systems after Application of Chlorhexidine to Dentin Contaminated with a Hemostatic Agent. J Dent (Shiraz). 2015;16(3):175-81.

10. Kuphasuk W, Harnirattisai C, Senawongse P, Tagami J. Bond strengths of two adhesive systems to dentin contaminated with a hemostatic agent. Oper Dent. 2007;32(4):399-405.

11. Kimmes NS, Olson TL, Shaddy RS, Latta MA. Effect of ViscoStat and ViscoStat Plus on composite shear bond strength in the presence and absence of blood. J Adhes Dent. 2006;8(6):363-6.

12. O'Keefe KL, Pinzon LM, Rivera B, Powers JM. Bond strength of composite to astringent-contaminated dentin using self-etching adhesives. Am J Dent. 2005;18(3):168-72.

13. Mohammadi N, Kimyai S, Bahari M, Pournaghi-Azar F, Mozafari A. Effect of aluminum chloride hemostatic agent on microleakage of class $\mathrm{V}$ composite resin restorations bonded with all-in-one adhesive. Med Oral Patol Oral Cir Bucal. 2012;17(5): e841-4.

14. Melkumyan TV, Kakhkharova DJ, Dadamova AD, Kamilov NKh, Siddikova SSh, Rakhmatullaeva ShI, Masouleh SM. Comparative Analysis of in vitro Performance of Total-Etch and Self-Etch Adhesives. International Journal of Biomedicine. 2016;6(4):283-6. doi: 10.21103/Article6(4)_OA7.

15. Terry DA. An essential component to adhesive dentistry: the rubber dam. Pract Proced Aesthet Dent. 2005;17(2):106, 108. 